\title{
Study on the Characteristics of Gut Microbiota in Chronic Hepatitis B Patients with Damp Heat Syndrome and Liver Depression and Spleen Deficiency Syndrome
}

\section{Kaiping Jiang ( $\nabla$ jkpingfs@126.com )}

Foshan Hospital of Traditional Chinese Medicine区Affiliated to Guangzhou University of Chinese Medicine https://orcid.org/0000-0002-1983-2259

\section{Qunfang Jiang}

Foshan Hospital of Traditional Chinese Medicine『Affiliated to Guangzhou University of Chinese Medicine

\section{Xiaoai Mo}

Foshan Hospital of Traditional Chinese Medicine『Affiliated to Guangzhou University of Chinese Medicine

\section{Jianhong Li}

Chongqing City Hospital of Traditional Chinese Medicine

\section{Hongtao Hu}

Foshan Hospital of Traditional Chinese Medicine『Affiliated to Guangzhou University of Chinese

Medicine

\section{Qinghua Huang}

Foshan Hospital of Traditional Chinese Medicine,Affiliated to Guangzhou University of Chinese

Medicine

\section{Wenqiang Guo}

Foshan Hospital of Traditional Chinese Medicine『Affiliated to Guangzhou University of Chinese Medicine

\section{Tengyu Qiu}

Foshan Hospital of Traditional Chinese Medicine,Affiliate to Guangzhou University of Chinese Medicine Jian Ren

Foshan Hospital of Traditional Chinese Medicine,Affiliated to Guangzhou University of Chinese Medicine

\section{Lei Zhang}

Foshan Hospital of Traditional Chinese Medicine区Affiliate to Guanghzou University of Chinese Medicine

\section{Youqing Xie}

Foshan Hospital of Traditional Chinese Medicine,Affiliate to Guangzhou University of Chinese Medicine 
Foshan Hospital of Traditional Chinese Medicine『Affiliated to Guangzhou University of Chinese Medicine

\section{Research}

Keywords: Chronic Hepatitis B (CHB), Intestinal Flora, Traditional Chinese Medicine (TCM), Damp Heat Syndrome, Liver Depression and Spleen Deficiency Syndrome

Posted Date: July 27th, 2020

DOI: https://doi.org/10.21203/rs.3.rs-45025/v1

License: (c) (i) This work is licensed under a Creative Commons Attribution 4.0 International License. Read Full License 


\section{Abstract}

Background: To explore the potential biological characteristics of traditional Chinese medicine(TCM) syndromes in CHB patients is of great significance to improve the level of TCM treatment. This study was designed to explore the characteristics of gut microbiota in $\mathrm{CHB}$ patients with two most common TCM Syndromes.

Methods: According to the diagnostic criteria of Western medicine, TCM and screening exclusion criteria, 65 cases of CHB patients with damp heat syndrome and 28 cases of CHB patients with liver depression and spleen deficiency syndrome were finally included in the study. All the basic information was gathered and the fresh fecal samples were collected for 16S rDNA sequencing. 16S rDNA of gut microbiota was sequenced using Illumina hiseq 2500 high-throughput sequencing platform.Based on the optimized sequence, OTU clustering analysis and taxonomic annotation were carried out.

Results: The difference in relative abundance of gut microbiota was significant between damp heat syndrome and liver depression and spleen deficiency syndrome in CHB patients. Cyanobacteria was only found in damp heat syndrome.The relative abundance of Erysipelotrichia and Subdoligranulum were higher in liver depression and spleen deficiency syndrome,while the relative abundance of Rhodospirillales, Alphaproteobacteria,and Lachnospira were higher in the damp heat syndrome.LEfSe analysis showed that Lachnospira,Olsenella and Subdoligranulum had significant difference in species among the two TCM syndromes.

Conclusions: The different characteristics of gut microbiota in the two TCM syndromes of CHB patients may play an important role in syndrome formation of TCM,which provides a new field of vision for the accurate diagnosis and treatment of TCM.

\section{Background}

Chronic hepatitis B virus (HBV) infection,which causes nearly one million deaths each year,remains a major public health problem worldwide. ${ }^{1,2}$ A modelling study estimated that the global prevalence of HBsAg was $3.9 \%$ in $2016 .^{2}$ Among the untreated patients with chronic hepatitisB virus infection, $15-40 \%$ progress to cirrhosis that may lead to liver failure and liver cancer. ${ }^{3}$ The development and prognosis of HBV infection-related diseases are closely associated with their complex immune response and virus interaction. A large amount of evidence reveals that the occurrence and development of chronic HBV infection is not only related to virus load, but also closely associated with immune response andgut microbiota. ${ }^{4}$ The host immune response induced by HBV infection not only drives the progress of the disease, but also significantly affects the effect of antiviral therapy. On the contrary, immune tolerance and ineffective response to HBV will lead to the continuous replication of HBV, thus developing into chronic infection. ${ }^{5}$ At present, it is considered that the exhaustion of T cells make the immune response of the body unable to start effective immune response to eliminate HBV, which leads to the continuous infection of the virus. ${ }^{6,7}$ The long-term effect of high load viral antigens in chronic HBV infection is the 
most direct reason for the depletion of T cells. Due to immature immune system of infants, about $90 \%$ of children's diseases become a chronic progression after HBV infection because of insufficient immune response, and the probability of chronic development will gradually reduce after 5 years old. In addition, about $25 \%$ of children can develop cirrhosis after HBV infection, which is just in line with the age of mature immune system and establishment of perfect intestinal microecology. 8,9

For the treatment of $\mathrm{CHB}$, even the most widely used nucleoside analogues or interferon can not achieve the cure of the disease. ${ }^{10,11}$ The above mentioned therapies of $\mathrm{CHB}$ to restore the immune function of the body and improve the T cell response may be an important target for the treatment of $\mathrm{CHB}$ in the future. There are many immunotherapies at present, but the toxic and side effects of its immune preparations, technology, cost etclimit the implementation of its research. As a research hotspot in the scientific community, gut microbiota has been proved to be closely related to the occurrence and development of some diseases of the human system. The gut microbiota can not only promote the maturity of the human immune system, but also affect the occurrence, development and prognosis of liver disease. ${ }^{12}$ The study of "Gut-liver Axis" shows that the liver and intestine are connected through portal vein and biliary tract system. ${ }^{13,14}$ The late studies have proved that the gut microbiota plays an important role in the development and prognosis of chronic HBV infection. For example, Wang's study has confirmed that the intestinal bacteria will shift after the alteration of gut microbiota, which will cause liver inflammation after entering the liver, and affect the immune function of liver $T$ cells and reduce the ability of clearing HBV at the same time. ${ }^{15}$ Therefore, it is of great significance to study the regulation of gut microbiota in the treatment of CHB.

The traditional Chinese medicine (TCM ) has been widely used in the treatment of CHB in China. ${ }^{16}$ Syndrome differentiation and treatment is the core content of traditional Chinese Medicine.It is necessary to find the potential biological characteristics of TCM Syndromes of chronic hepatitis B to improve the level of TCM clinical syndrome differentiation and treatment. ${ }^{17}$ TCM has also been proved to have a certain effect in regulating gut microbiota,so the research of TCM syndromes based on gut microbiota can not only enrich the scientific connotation of TCM syndromes, but also provide a new direction for the theoretical research of TCM. ${ }^{18}$ The damp heat syndrome and the liver depression and spleen deficiency syndrome are the most common TCM syndromes in patients with CHB. ${ }^{19,20.21}$ By analyzing the characteristics of gut microbiota in $\mathrm{CHB}$ patients with damp heat syndrome and liver depressione and spleen deficiency syndrome, the biological connotation of the two TCM syndromes was discussed in this study.

\section{Methods}

\section{Sample Collection}

This study was approved by the medical ethics committee of Foshan Hospital of traditional Chinese medicine.A total of 157 patients with HBV infection (group AA)and 35 healthy people(group JK) who were 
come from Foshan Hospital of TCM from April 2018 to January 2019 were enrolled in this study and signed written informed consent. All these patients were diagnosed according to the EASL 2017 Clinical Practice Guidelines on the Management of Hepatitis B Virus Infection and the Guideline of Prevention and Treatment for Chronic Hepatitis B (2015 Update):22,23(1)historical history of CHB or HBsAg positive for more than 6 months;(2)ages 18 to 65 ;(3) $2 \times$ ULN $\leq$ ALT $\leq 10 \times$ ULN,and TBIL $\leq 51.3 \mathrm{umol} / \mathrm{L}$;(4)HBVDNA level ( $\geq 2000 \mathrm{IU} / \mathrm{ml}$ ); (5)anormal testing result of liver color doppler ultrasound and transient elastography(FibroScan).The exclusion criteria of CHB patients were as follows: (1) suffering from disease of the kidney, heart, brain, or lung, or any other organ;(2) suffering from alcoholic and nonalcoholic fatty liver disease,chronic severe hepatitis, cirrhosis or liver tumor; (3) pregnant or lactating women; (4) with special dietary habits, such as vegetarians;(5) those who have used antibiotics and microecological agents in the past three months. The TCM syndrome diagnosis was carried out according to the standards of traditional Chinese medicine syndrome differentiation for viral hepatitis: ${ }^{24}$ (1) damp heat syndrome; (2) liver depression and spleen deficiency syndrome; (3)blood stasis syndrome; (4) Yin deficiency of liver and kidney syndrome; (5) Yang deficiency of spleen and kidney.In order to ensure the reliability of TCM syndrome differentiation, the information collection of TCM four diagnoses of all patients was carried out by the intelligent diagnosis instrument (SMF-II TCM comprehensive diagnosis system, produced by Wuhu shengmeifu Technology Co., Ltd.). All the study cases enrolled was shown in the flow chart(Fig. 1).

\section{Demographic and Clinical Characteristics}

In 157 patients (group AA), there were 46 males and 19 females, with an average age of $33.05 \pm$ 7.42 years. There were 20 males and 15 females with an average age of $34.14 \pm 8.85$ years in the healthy control group (group JK). There was no significant difference in gender and age between the two groups $(P>0.05)$. Among the 65 cases of $\mathrm{CHB}$ patients with damp heat syndrome (group $G$ ), there were 46 males and 19 females with an average age of $32.68 \pm 6.59$ years. There were 28 cases of CHB patients with liver depression and spleen deficiency (group HJ), 14 male and 14 female, with an average age of $32.11 \pm$ 7.75 years. There was no significant difference in gender and age between the two TCM syndrome groups $(P>0.05)$.In the two TCM syndrome groups of CHB patients, ALT, AST, TBIL and HBV DNA etc diagnostic indicators were also observed(Table 1). 
Table 1

Demographic and clinical characteristics in this study

\begin{tabular}{|c|c|c|c|c|}
\hline & $\mathrm{AA}(\mathrm{n}=157)$ & $J K(n=35)$ & $G(n=65)$ & $H J(n=28)$ \\
\hline Age & $16 \sim 57$ & $24 \sim 55$ & $16 \sim 53$ & $17 \sim 47$ \\
\hline means $\pm S D$ & $33.05 \pm 7.42$ & $34.14 \pm 8.85$ & $32.68 \pm 6.59$ & $32.11 \pm 7.75$ \\
\hline \multicolumn{5}{|l|}{ Sex } \\
\hline Female & $64(41 \%)$ & $15(43 \%)$ & $19(29 \%)$ & $14(50 \%)$ \\
\hline Male & $93(59 \%)$ & $20(57 \%)$ & $46(71 \%)$ & $14(50 \%)$ \\
\hline \multicolumn{5}{|l|}{ ALT(U/L) } \\
\hline means \pm SD & & & $135.42 \pm 136.89$ & $84.60 \pm 107.59$ \\
\hline \multicolumn{5}{|l|}{ AST(U/L) } \\
\hline means \pm SD & & & $103.26 \pm+275.43$ & $43.93 \pm 32.77$ \\
\hline \multicolumn{5}{|l|}{ GGT(U/L) } \\
\hline means $\pm S D$ & & & $47.95 \pm 43.99 *$ & $28.93 \pm 25.04$ * \\
\hline \multicolumn{5}{|c|}{ HBV DNA(IU/mL) } \\
\hline means $\pm S D$ & & & $6.76 \pm 1.51$ & $6.71 \pm 1.48$ \\
\hline \multicolumn{5}{|c|}{$\begin{array}{l}\text { NOTE.AA:patients with HBV infection JK:healthy people G: damp heat syndrome of CHB;HJ:liver } \\
\text { depression and spleen deficiency syndrome of CHB }\end{array}$} \\
\hline \multicolumn{5}{|c|}{ ALT: alanine transarninase;AST: aspartate aminotransferase;GGT : $y$-glutamyl transpeptidase } \\
\hline
\end{tabular}

\section{Data Processing and Bioinformatics Analysis}

Fresh fecal samples were collected from all participants, frozen immediately and stored at $-80^{\circ} \mathrm{C}$ for further processing. Fecal DNA extraction and 16S rDNA gene sequencing were completed by Institute of Microbiology, Chinese Academy of Sciences.In this experiment, a double-index double amplification method was used to prepare a PCR amplicon library. The primers for the first PCR amplification were obtained by adding partial sequencing adapter sequences at both ends of the universal primer for specific amplification to complete specific PCR amplification. At the same time, a part of the adapter sequence was added.The forward and reverse primers of the second amplification each had an 8-base index sequence.The resulting PCR product included the target sequence and a complete sequencing adapter sequence with two indexes.Environmental microorganism DNA samples were subjected to the first PCR amplification with universal primers (for example, 357F / 806R for 16SV3V4 region sequencing).Beads were purified and a second amplification was performed after purification to ensure that each sample had a different index combination. After the library was quantified and homogenized, 
samples with different index sequences were mixed together. The mixed samples were lllumina Hiseq. The library was sequenced by 250 bp (250PE) double-ended library using 2500 high-throughput sequencing platform. The offline data was split using different index sequences.Based on the overlap relationship,the PE reads was obtained by Hiseq sequencing. The obtained double-end sequences were spliced into a target region sequence,and the target sequence was subjected to quality control filtering.

The filtered sequence was compared with a reference database, and the chimeric sequence was removed to obtain the final optimized sequence.OTU clustering analysis and Annotation of Taxonomy were based on the optimized sequence.Analysis of diversity index was based on OTU clustering results.Analysis of species structure and species difference were based on taxonomic information.

\section{Statistical Analysis}

The continuous variables were summed up as mean and standard deviation, the discrete variables were expressed as number and percentage, and compared with Chi-square test. All the analyses were carried out by SPSS 20.0 software, $P<0.05$, with statistical significance.

\section{Results}

\section{Gut Microbiota Communities in Patients with HBV Infection and Healthy People}

The LEfSe analysis revealed that several gut microbiota were identified as being significantly different among the two groups.According to LEfSe analysis, the Prevotellaceae,Veillonellaceae,Selenomonadales and Negativicutes were more abundant in the intestine of patients with HBV infection(Fig. 2A,2B). The gut microbiota of healthy people was enriched with Desulfovibrionaceae, Desulfovibrionales, Deltaproteobacteria, Enterobacteriaceae, Enterobacteriales, Verrucomicrobiaceae, Verrucomicrobiales and Verrucomicrobiae(Fig. 2A,2B).

\section{Changes of Gut Microbiota in CHB Patients with Damp Heat Syndrome and Liver Depressione and Spleen Deficiency Syndrome}

The Veen diagram reflecting the difference between damp heat syndrome(group $\mathrm{G}$ ) and liver depression and spleen deficiency syndrome(group HJ)was depicted(Fig. 3A). A total of 858 OTUs were identified in all samples, including 845 OTUs in damp heat syndrome(group G)and 833 OTUs in liver depression and spleen deficiency syndrome(group HJ)(Fig. 3A).Among them, 820 OTUs existed in both groups. Partial least squares discriminant analysis (PLS-DA) and principal component analysis (PCA) were used to calculate the $\beta$ diversity of each group to describe the composition difference between the two TCM syndromes. PCA and PLS-DA analysis showed that the sample points were almost totally separated but 
appeared partially overlapped.The PLS-DA results showed that the gut microbiota of subjects in CHB patients with damp heat syndrome and liver depression and spleen deficiency syndrome could be distinguished at the OTU level(Fig. 3B).So the composition of gut microbiota in CHB patients with the two TCM syndromes were significantly different at the OTU level.

The relative abundances of the top 15 bacteria in two TCM Syndromes of CHB patients were compared at the level of phylum, category, order, family and genus. The majority of sequences were arranged in four bacterialphyla (i.e.Firmicutes,Bacteroidetes,Actinobacteria,Proteobacteria) (Fig. 4A).Among them,Firmicutes was the most,and the second dominate phyla was Bacteroidetes.At the phylum level, Cyanobacteria was only detected in damp heat syndrome(group G).In addition,there was a great significant difference in the relative abundance of Erysipelotrichia, Alphaproteobacteria, Rhodospirillales, Erysipelotrichaceae, Rhodospirillaceae, Lachnospiraand, Subdoligranulum between the two TCM syndromes(group G and group HJ) (Table 2).

Table 2:

Relative abundance of gut microbiota in CHB patient with damp heat syndrome and liver depression and spleen deficiency syndrome

(mean \pm SD)

\begin{tabular}{|lll|}
\hline Gut microbiota & Group G & Group HJ \\
\hline Erysipelotrichia & $3.234 \pm 1.704$ & $4.386 \pm 3.061^{*}$ \\
\hline Alphaproteobacteria & $0.025 \pm 0.092$ & $0.001 \pm 0.004^{*}$ \\
\hline Rhodospirillales & $0.024 \pm 0.092$ & $0.001 \pm 0.004^{*}$ \\
\hline Erysipelotrichaceae & $3.234 \pm 1.704$ & $4.386 \pm 3.061^{*}$ \\
\hline Rhodospirillaceae & $0.025 \pm 0.092$ & $0.001 \pm 0.004^{*}$ \\
\hline Subdoligranulum & $1.159 \pm 1.119$ & $1.923 \pm 1.324^{*}$ \\
\hline Lachnospira & $2.787 \pm 2.187$ & $1.530 \pm 1.108^{*}$ \\
\hline NOTE. $* P<0.05 ; G:$ damp heat syndrome;HJ: liver depression and spleen deficiency syndrome. \\
\hline
\end{tabular}

The composition of the intestinal bacteria in the two groups was compared using the LEfSe analysis.According to LEfSe analysis,the Lachnospira was more abundant in damp heat syndrome(group G), while the Olsenella and Subdoligranulum were more abundant in liver depression and spleen deficiency syndrome(group HJ)(Fig. 5).

\section{Discussion}


A large number of previous studies have confirmed that there are different degrees of alteration of gut microbiota in all kinds of chronic liver diseases, and the changes of the gut microbiota play an important role in the occurrence and development of chronic liver disease. ${ }^{25}$ Moreover, treating a disease or improving its prognosis can be achieved by regulating the gut microbiota. Some studies have shown that partial beneficial bacteria may play a role in the immune clearance of viruses in young HBV infected persons. Another study found that the fecal bacteria transplantation in $\mathrm{CHB}$ patients with HBeAg positive was more likely to produce HBeAg seroconversion than patients with oral antiviral drugs alone. ${ }^{26}$ In addition, The regulatory effect of traditional Chinese medicine on gut microbiota has always been concerned. Ever-increasing evidence suggests that gut microbiota plays a crucial role in Herbal medicine therapy by complicated interplay with Herbal medicine components. ${ }^{27}$ It is of great significance to study the biological basis of TCM syndrome based on gut microbiota to guide the syndrome differentiation and treatment of CHB.

In this study, the TCM Syndromes of 116 patients with CHB showed damp heat syndrome in 65 cases (56.03\%), liver depressione and spleen deficiency syndrome in 28 cases $(24.14 \%)$, blood stasis syndrome in 10 cases (8.62\%), Yin deficiency of liver and kidney syndrome in 9 cases (7.76\%), and Yang deficiency of spleen and kidney in 4 cases (3.45\%), which further confirmed that damp heat syndrome and liver depressione and spleen deficiency syndrome were the main TCM Syndromes of CHB. It should be noted that damp heat syndrome was the most common TCM syndromes in CHB patients, which might be related to the theory of traditional Chinese medicine that damp heat was the basic pathological factor of CHB. ${ }^{28}$ Except for the slightly different GGT changes, there was no significant difference in ALT, AST, and HBV DNA etc major laboratory diagnostic indexes between damp heat syndrome and liver depressione and spleen deficiency syndrome $(P>0.05)$. Therefore, it was necessary to explore the biological indicators that can further reflect the scientific connotation of these two syndromes.

The analysis of Venn diagram of gut microbiota showed that there was a large number of the same OTUs in the two TCM syndrome groups, and there were also specific OTUs in each group.In PLS-DA analysis, the flora composition of the two groups was clearly separated, and there were overlaps in the middle. All of these indicated that there were differences in the gut microbiota of CHB patients with damp heat syndrome and liver depression and spleen deficiency syndrome, but the differences could not be completely distinguished. Why did this happen?The possible explanation was as follows:According to the theory of TCM,damp heat is the basic pathological factors of $\mathrm{CHB}$, which can run through the whole course of the disease.Therefore, the liver depression and spleen deficiency syndrome can not only contain the syndrome factors of damp heat syndrome in part,but also can but also can transform from liver depression and spleen deficiency to damp heat,due to spleen deficiency can't transport and change water dampness with a result that a prolonged water dampness will accumulated into damp heat. ${ }^{28}$

Based on the analysis of relative abundance of gut microbiota at the level of phylum, class, order, family and genus, it showed that only Cyanobacteria at the level of phylum was found in the damp heat syndrome of $\mathrm{CHB}$ patients.Cyanobacteria may produce microcystin which can cause liver damage and 
necrosis, even induce the occurrence of liver cancer. In addition, it may also have a significant inhibitory effect on immune function. ${ }^{29,30}$ Sowe should pay attention to the role of Cyanobacteria in the occurrence and development of damp heat syndrome in CHB patients. In addition, the relative abundance of Subdiligranulum was lower in liver depression and spleen deficiency syndrome in our study.It had been found that the abundance of Subdiligranulum in the HBV decompensate cirrhosis patients was significantly lower than that in the healthy people. ${ }^{31}$ Whether "liver depression and spleen deficiency syndrome of CHB - Subdiligranulum - decompensation of HBV related cirrhosis " has a certain inevitable relationship needs further study.

Furthermore, the relative abundance of Erysipelotrichia and Erysipelotrichaceae were higher in liver depression and spleen deficiency syndrome.Previous studies had shown that erysipelas were not only positively correlated with fatty liver, ${ }^{32}$ but also strongly correlated with diabetic nephropath. ${ }^{33}$ Subdoligranulum,as a biomarker in liver depression and spleen deficiency syndrome,the changes of its abundance will lead to metabolic disorders of the host. ${ }^{34}$ In addition, this part of research showed that the number of Subdolegranulum in patients with ulcerative colitis was higher than that in healthy people. Therefore, Subdoligranulum may be related to the intestinal inflammation. The intestinal inflammation, intestinal mucosal barrier damage, harmful intestinal bacteria and their metabolites enter the liver through the portal vein, and then promote the liver inflammation or aggravate the liver inflammation. ${ }^{35}$ Therefore, CHB patients with liver depression and spleen deficiency syndrome may be more likely to develop fatty liver, diabetes and other metabolic diseases than those with damp heat syndrome. This study only conducted a preliminary study on the characteristics of gut microbiota of the two most common TCM syndromes in patients with CHB, the sample size was not large enough and the study of metabolites and related metabonomics of gut microbiota was still blank. In particular, the interpretation of the biological significance of related gut microbiota in TCM needs to be further studied.

\section{Conclusion}

There is a significant difference in gut microbiota between damp heat syndrome and liver depression and spleen deficiency in CHB patients.These characteristics of gut microbiota may be to guide us to better understanding of the biologic basis of the TCM syndrome and standardization of syndrome classification in CHB patients, which in turn improve the overall level of TCM diagnosis and treatment of $\mathrm{CHB}$ and open up a new field of vision for the selection of TCM compound treatment targets.

\section{Abbreviations}

CHB: Chronic hepatitis B; TCM: Traditional Chinese medicine; HBV: Chronic hepatitis B virus; group AA: Patients with HBV infection; group JK: Healthy people; group G: CHB patients with damp heat syndrome; group HJ: CHB patients with liver depression and spleen deficiency syndrome;OUT:Optical transform unit;ALT: Alanine aminotransfease; AST: Aspartate transaminase; GGT: Glutamyl transpeptidase; PLS-DA: 
Partial least squares discriminant analysis; PCA: principal component analysis; LEfSe analysis :Linear discriminant analysis Effect Size.

\section{Declarations}

\section{Acknowledgments}

In this study, the detection and analysis of gut microbiota was greatly supported and helped by teacher Luna from the Institute of Microbiology, Chinese Academy of Sciences.

\section{Authors' contributions}

Kaiping Jiang conceived and designed the study,collected and analyzed the large sample data and completed the writing of the paper. Qunfang Jiang and Xiaoai Mo carried out the observation of clinical cases,the data induction,clinical trial registration and some writing of the paper, contributed equally to this work. Jianhong Li carried out the observation of clinical cases and the part of the clinical trial registration. Hongtao Hu carried out the observation of clinical cases. Qinghua Huang $\unrhd a n d ~ K a i z h o u$ Huang collected clinical specimen. Wenqiang Guo,Tengyu Qiu, and Jian Ren carried out the observation of Clinical cases. Youqing Xie, and Lei Zhang carried out the literature search and data extraction.

\section{Funding}

Foshan High Level Medical Key Discipline Construction Project and "Peak Climbing Plan" for Foshan High Level Hospital Construction囚and the Sixth Group of National Senior Chinese Medicine Experts' Academic Experience InheritanceProject.

\section{Availability of data and materials}

Most of the data in the present study are included in this article and the original data can be obtained through the corresponding author .

\section{Ethics approval and consent to participate}

The study protocol was approved by the Medical Ethics Review Committee for Foshan Hospital of Traditional Chinese Medicine (Ethical Review NO.『2018®029)

\section{Consent to publish}

Not applicable. 


\section{Competing interests}

The authors declare that they have no competing interests.

\section{Conflict of interest statement}

The authors have declared that no conflict of interest exists.

\section{References}

1. Schweitzer A, Horn J, Mikolajczyk R T, et al. Estimations of worldwide prevalence of chronic hepatitis B virus infection: a systematic review of data published between 1965 and 2013. Lancet 2015;386:1546-1555.

2. Nayagam S, Thursz M, Sicuri E, et al. Requirements for global elimination of hepatitis B:amodelling study. Lancet Infect Dis 2016;16:1399-1408.

3. Lydia S Y Tang, Emily Covert, Eleanor Wilson, et al. Chronic Hepatitis B Infection: A Review. JAMA 2018;319:1802-1813.

4. Xu D, Huang Y, Wang J. Gut microbiota modulate the immune effect against hepatitis $B$ virus infection. Eur J Clin Microbiol Infect Dis 2015;34:2139-2147.

5. Fu-Sheng Wang, Zheng Zhang. Host immunity influences disease progression and antiviral efficacy in humans infected with hepatitis B virus. Expert Rev Gastroenterol Hepatol 2009;3:499-512 .

6. Cheng Sun, Peixiang Lan, Qiuju Han , et al. Oncofetal gene SALL4 reactivation by hepatitis B virus counteracts miR-200c in PD-L1-induced T cell exhaustion. Nat Commun 2018;9:1241.

7. Paola Fisicaro, Carolina Boni , Valeria Barili , et al. Strategies to overcome HBV-specific T cell exhaustion: checkpoint inhibitors and metabolic re-programming. Curr Opin Virol 2018;30:1-8.

8. Liang, T J. Hepatitis B: the virus and disease. Hepatology ;49(Supplement S5).

9. Karsten Wursthorn, Heiner Wedemeyer, Michael P Manns. Managing HBV in patients with impaired immunity. Gut 2010;59:1430-1445.

10. Yuanyuan Kong, Hong You, Jidong Jia. Oral antiviral therapy reduces the risk of hepatocellular carcinoma in persons with chronic hepatitis B infection: combining evidence and common sense. Hepatol Int 2016;10:239-241.

11. Massimo Levrero, Teresa Pollicino, Jorg Petersen, et al. Control of cccDNA function in hepatitis B virus infection. J Hepatol 2009;51:581-592.

12. Amit Goel, Mahesh Gupta, Rakesh Aggarwal. Gut microbiota and liver disease. J Gastroenterol Hepatol 2014;29:1139-1148.

13. Jian-Wen Jiang, Xin-Hua Chen , Zhigang Ren, et al. Gut microbial dysbiosis associates hepatocellular carcinoma via the gut-liver axis. Hepatobiliary Pancreat Dis Int 2019;18:19-27. 
14. Marco Poeta, Luca Pierri, Pietro Vajro. Gut-Liver Axis Derangement in Non-Alcoholic Fatty Liver Disease. Children (Basel) 2017;4:66.

15. Wang JZ, Zhu QF, Xia P, et al. Study on the mechanism of intestinal flora influencing the outcome of HBV infection. Abstracts of the 12th national academic congress of immunolog.2017.

16. Chen Y, Zhu J. Anti-HBV effect of individual traditional Chinese herbal medicine in vitro and in vivo: An analytic review. J Viral Hepat $2013 ; 20: 445-452$.

17. Li Ma, Xiuli Zheng, Yu Yang 1, et al. Epigenetic differences of chronic hepatitis B in different TCM syndromes: Protocol for a case-control, non-interventional, observational clinical study. Medicine (Baltimore) 2018;97:e12452 .

18. Wu Jiajia , Li Xiaojuan, Chen Jiaxu. Review of Study on Correlation between Intestinal Microecology and Traditional Chinese Medicine Syndrome [in Chinese]. Journal of Traditional Chinese Medicine2018;59(14):1247-1251.

19. Zheng Xiuli, Feng Quansheng, Jia Rui, et al. The syndrome pattern distribution and synchronous quality of life of 692 chronic hepatitis B patients [in Chinese]. Lishizhen Medicine and Materia Medica Research 2014;(2):513-514.

20. Ye Yong'an, Tian Delu,Jiang Jian,et al. Study on Common Symptoms and Distribution Characteristics Of Traditional Chinese Medicine Syndromes of 1003 Chronic Hepatitis B(ALT $\geq 2 U L N)$ Patients [in Chinese].WORLD CHINESE MEDICINE 2015;10(9):1305-1308.

21. Mao Guo, Cai.Guangxian,Chen Bin.The TCM Syndrome Distribution Characteristic of 1868 Chronic Hepatitis B Patients and the Correlation [in Chinese]. Journal of Hunan University of Chinese Medicine 2014;34(4):24-28.

22. European Association for the Study of the Liver. EASL 2017 Clinical Practice Guidelines on the Management of Hepatitis B Virus Infection. J Hepatol 2017;67:370-398.

23. Jinlin Hou, Guiqiang Wang , Fusheng Wang, et al. Guideline of Prevention and Treatment for Chronic Hepatitis B (2015 Update). J Clin Transl Hepatol 2017;5:297-318.

24. Li Xiuhui, Yang Huasheng, Li Fengyi, et al. The standards of traditional Chinese medicine syndrome differentiation for viral hepatitis [in Chinese]. Journal of Clinical Hepatology 2017;33(10):17-24.

25. Herbert Tilg, Christoph Grander, Alexander R Moschen. How does the microbiome affect liver disease? Clin Liver Dis (Hoboken) 2016;8:123-126.

26. Han-Hsuan Chou, Wei-Hung Chien, Li-Ling Wu, et al. Age-related immune clearance of hepatitis B virus infection requires the establishment of gut microbiota. Proc Natl Acad Sci U S A 2015;112:21752180.

27. Jun Xu, Hu-Biao Chen, Song-Lin Li .Understanding the Molecular Mechanisms of the Interplay Between Herbal Medicines and Gut Microbiota. Med Res Rev 2017;37:1140-1185.

28. Kai-Ping Jiang, Jian-Hong Li, Hong-Tao Hu, et al. FibroScan vs hepatic histopathology for diagnosis of liver fibrosis in chronic hepatitis B patients with liver stagnation and spleen deficiency syndrome or damp and heat accumulation syndrome[in Chinese]. World Chinese Journal of Digestology 2014; 22 (19): 2768-2773. 
29. J Rapala1, K Sivonen, C Lyra, et al. Variation of Microcystins, Cyanobacterial Hepatotoxins, in Anabaena spp. as a Function of Growth Stimuli. Appl Environ Microbio/ 1997;63:2206-2212.

30. Carmichael WW. The Toxins of Cyanobacteria. Sci Am 1994;270:78-86.

31. Wang Yixuan, Li Wei, Cheng Danying, et al. Characteristics of intestinal flora of patients with hepatitis B related decompensated cirrhosis[in Chinese]. Chinese Journal of Experimental and Clinical Infectious Disease(Electronic Version) 2019;13(02):110-116.

32. Spencer MD, Hamp TJ, Reid RW, et al. Association Between Composition of the Human Gastrointestinal Microbiome and Development of Fatty Liver With Choline Deficiency. Gastroenterology 2011;140:976-986 .

33. Kikuchi K, Saigusa D, Kanemitsu Y, et al.Gut microbiome-derived phenyl sulfate contributes to albuminuria in diabetic kidney disease. Nat Commun 2019;10:1835.

34. Jason Lloyd-Price, Cesar Arze, Ashwin N Ananthakrishnan,et al. Multi-omics of the Gut Microbial Ecosystem in Inflammatory Bowel Diseases. Nature $2019 ; 569: 655-662$.

35. Mondot S,Kang S,Furet JP, et al. Highlighting new phylogenetic specificities of Crohn's disease microbiota. Inflamm Bowel Dis 2011;17:185-92.

\section{Figures}

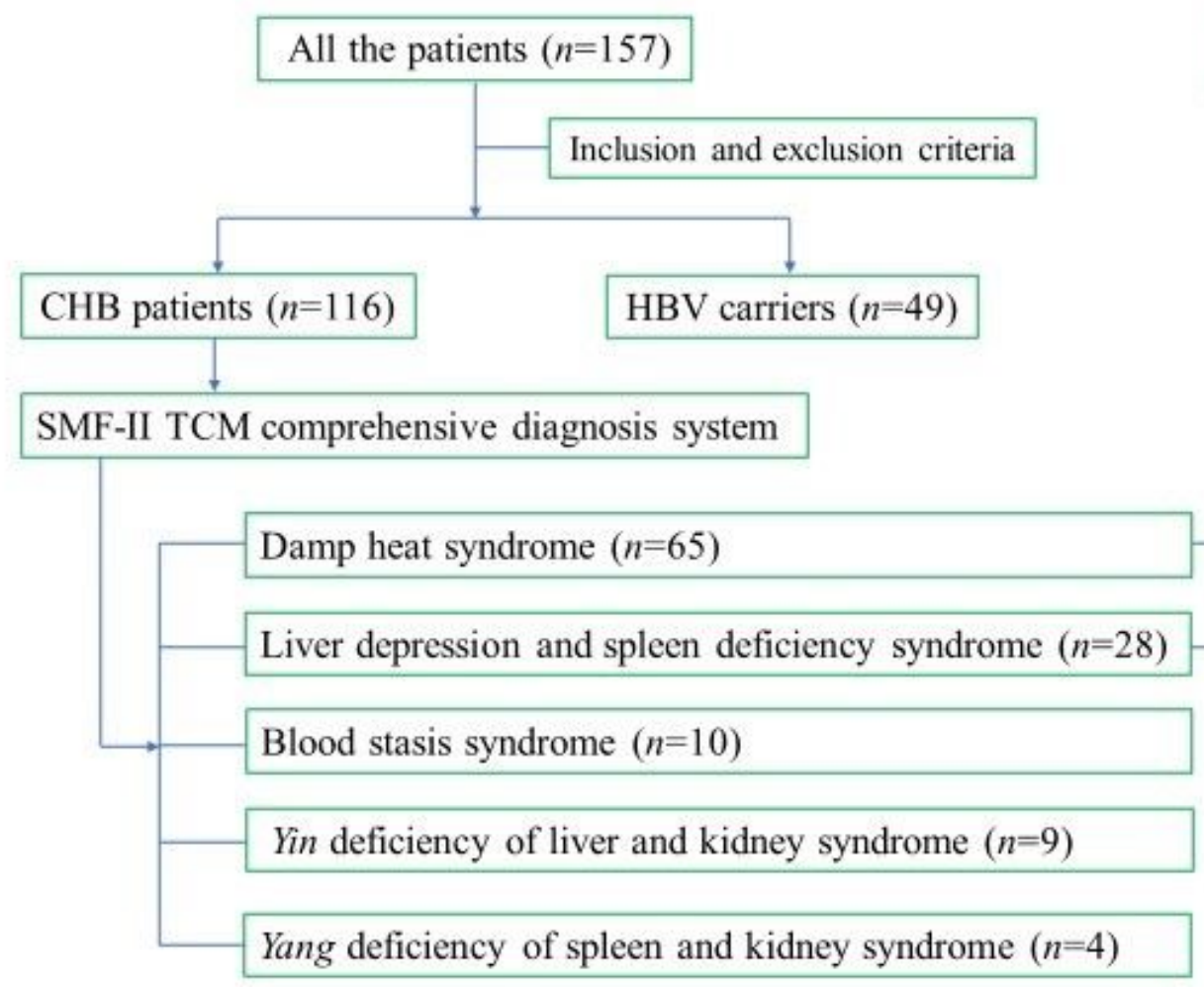

Healthy control group $(n=35)$

Analysis of the characteristics of gut microbiota based on $16 \mathrm{~S}$ rDNA gene sequencing

\section{Figure 1}

Study cases enrolled flow chart 
A

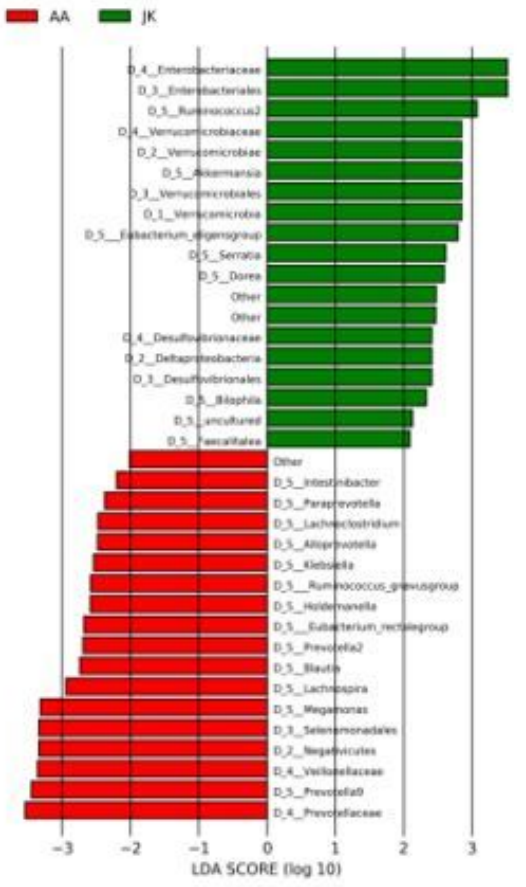

B

$\mathrm{AAA}$
$\mathrm{JK}$

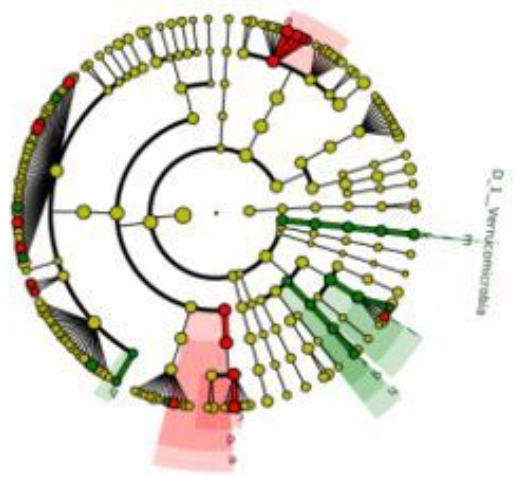

NOTE:Red:AA-enriched taxa-Green:JK-enriched taxa.JK: Healthy control; AA:CHB patients; LDA:Linear discriminant analysis.

\section{Figure 2}

A: Cladogram for taxonomic classification of gut microbiota B: Key microbiota contributing to the composition of gut microbiota in AA,and JK samples

A

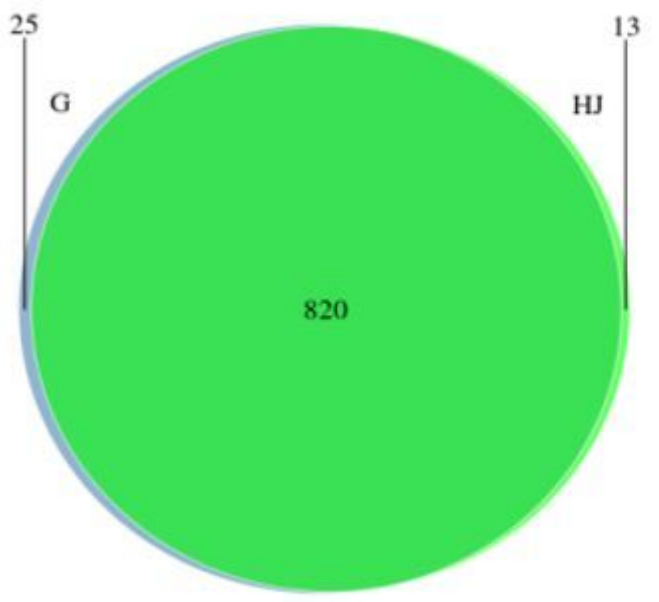

B

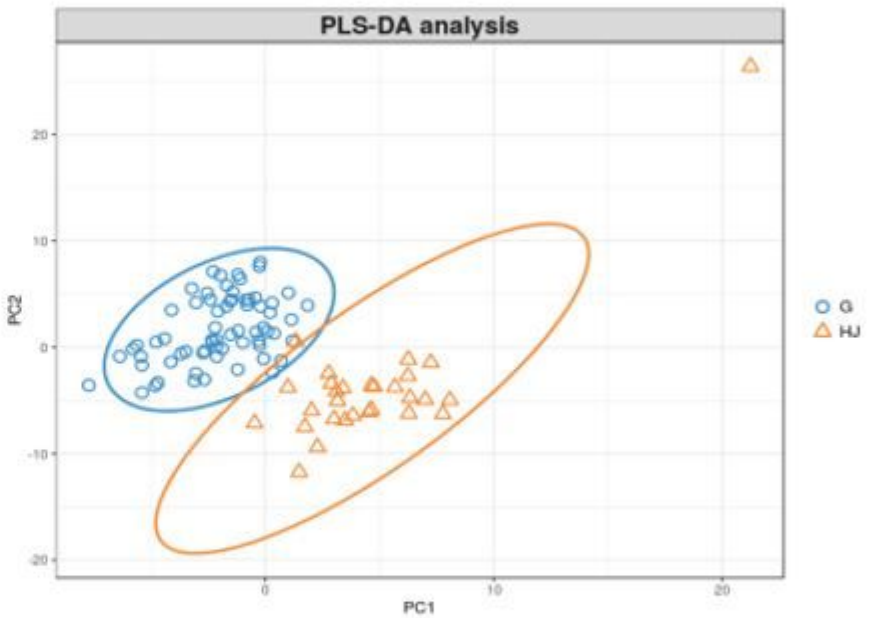

NOTE:; the red triangle represents each sample in the HJ group; the blue circle represents each sample in the G group(B). G: damp heat syndrome; HJ: the liver depression and spleen deficiency syndrome; OUT: operational taxonomic units. 
Figure 3

A: Venn diagram illustrating the overlap of OTUs identified in the gut microbiota from the two groups B: Partial least squares discriminant analysis (PLS-DA) of two groups
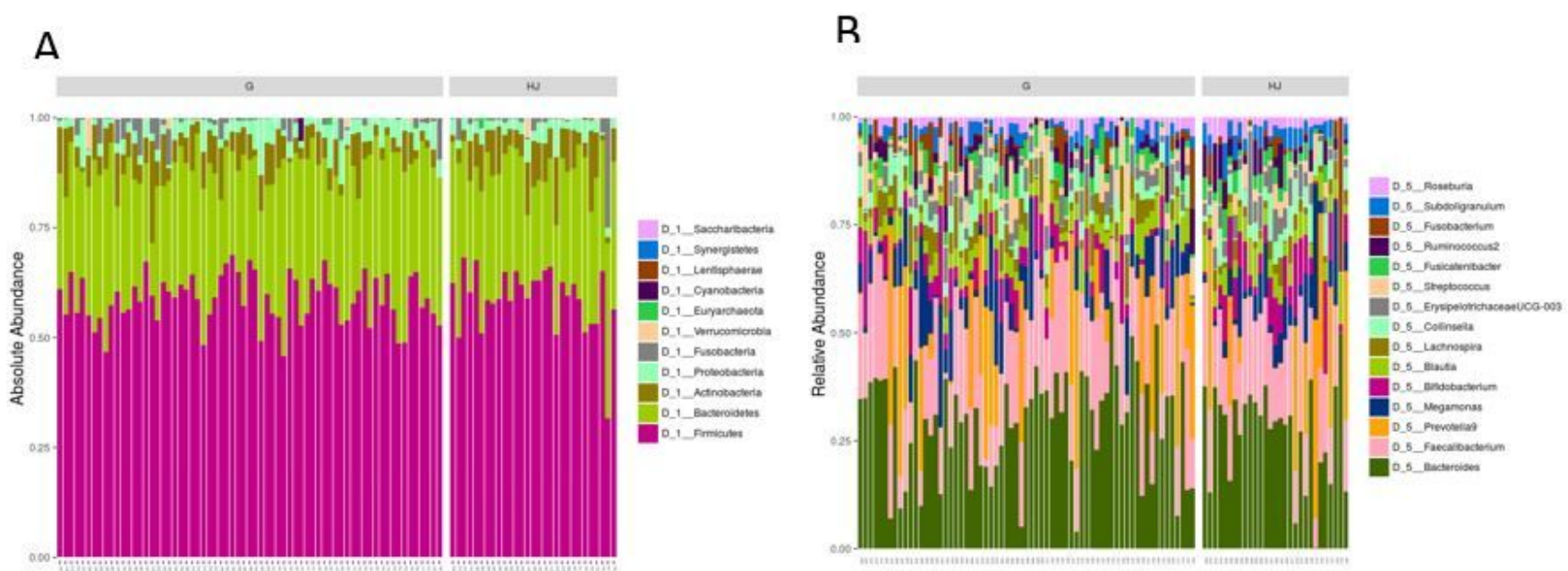

NOTE:G: damp heat syndrome; HJ: liver depression and spleen deficiencysyndrome.

\section{Figure 4}

A:The relative abundance of the gut microbiota phyla B: The relative abundance of the gut microbiota genera

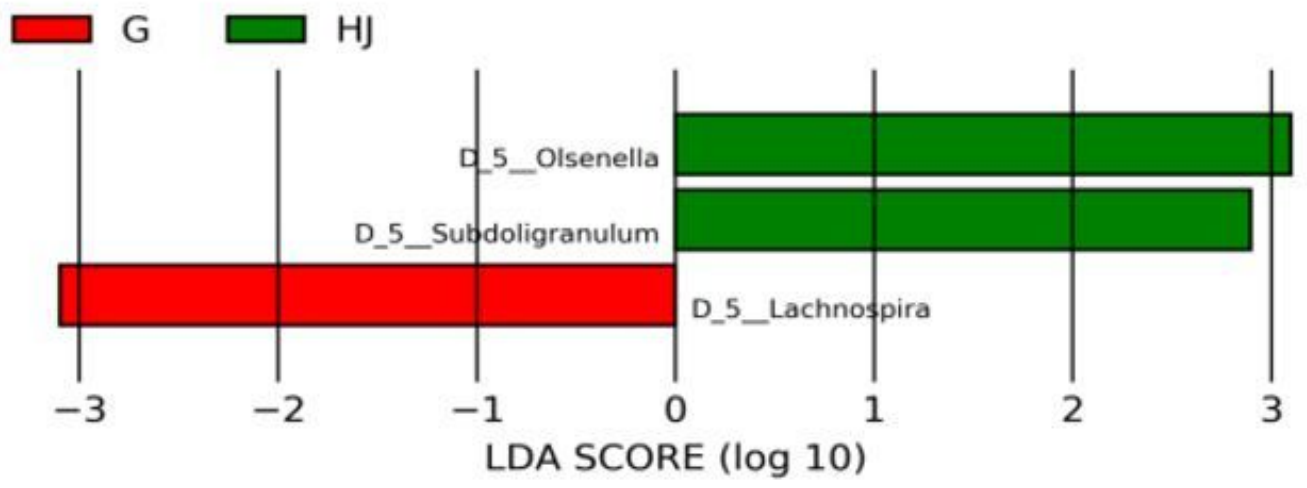

NOTE: G: Damp heat syndrome; HJ: The liver depression and spleen deficiency syndrome.

\section{Figure 5}

Key microbiota contributing to the composition of gut microbiota in $\mathrm{G}$,and $\mathrm{HJ}$ samples. 